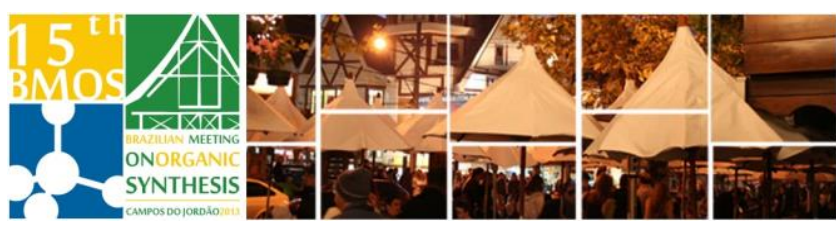

\title{
Synthesis of naphthoxazole derivates from lapachol and their isolation by countercurrent chromatography
}

\author{
Karina P. Del Rioํㅜ, Gilda G. Leitão ${ }^{1}$, Kelly C. Gallan de Moura ${ }^{1}$. \\ 1- Núcleo de Pesquisa de Produtos Naturais, CCS, Universidade Federal do Rio de Janeiro, 21944-970, Rio \\ de Janeiro - RJ. \\ karina.p.delrio@gmail.com
}

Keywords: lapachol, naphthoxazole, countercurrent chromatography

\section{INTRODUCTION}

In recent years, a number of studies showed that the heterofunctionalization of the quinone structure of $\beta$-lapachone in the preparation of bioactive imidazoles and oxazoles were of great value in the treatment of Chagas's disease. ${ }^{1,2}$

The use of other natural quinones, such as lapachol (1), in the synthesis of new heterocycles becomes important in the development of new bioactive substances, either in the combat of Chagas's disease or in the treatment of other diseases.

Silica gel column chromatography is the chromatographic method conventionally used for the purification of these derivates, however it consumes much time and large amounts of solvent. In order to improve the isolation procedure, high speed countercurrent chromatography was used instead. ${ }^{3}$

\section{RESULTS AND DISCUSSION}

Lapachol (1) was obtained by extraction of milled wood of Tabebuia $s p$. The heterocycles were synthesized by nucleophilic condensation of lapachol (1) with aldehydes which generated the most active imidazoles in previous studies with $\beta$ lapachone in the presence of ammonium acetate and acetic acid as solvent under reflux (Scheme 1).

The formation of products, as well as the end of the reactions were monitored by thin layer chromatography (TLC) using silica gel chromatographic plates where the main products were oxazole derivates together with byproducts of different polarities.

Each reaction lead to the formation of two products with very close polarities, which were purified by countercurrent chromatography (PC Inc.), using hexane:acetonitrile:methanol $(2: 2: 1)$ as solvent system, upper phase as mobile phase (normal phase) at a flow rate of $2 \mathrm{~mL} / \mathrm{min}$. The sample reaction (100 mg) was dissolved in $5 \mathrm{~mL}$ of the solvent system $(2,5 \mathrm{~mL}$ of each phase) and injected on a $80 \mathrm{~mL}$ column. 60 fractions of $4 \mathrm{~mL}$ were collected, and the substances retained on the column were recovered in the elution-extrusion mode (over 20 fractions) using the stationary phase as mobile phase.

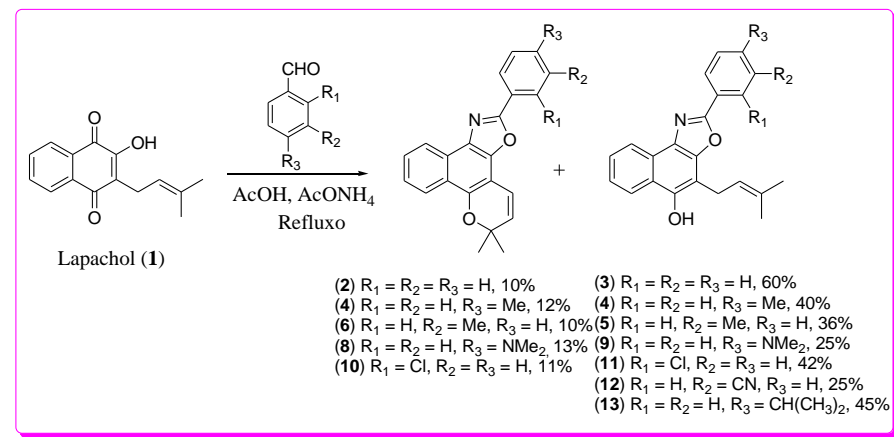

Scheme 1: Obtention of naphthoxales derivates.

Spectroscopic data analyses showed that one of the products was the naphtoxazole of $\beta$ xiloidone, and the other, the naphthoxazole with the isoprenyl side chain derived from lapachol (1).

\section{CONCLUSION}

The condensation reactions with lapachol (1) led to the formation of naphthoxazoles as major reaction products. High speed countercurrent chromatography proved to be an efficient technique for the separation and purification of these derivates. using the same solvent system, economy of time and solvent when compared to conventional liquid chromatography technique (with solid support).

\section{ACKNOWLEDGEMENTS}

from the CNPq.

This research was supported by grants REFERENCES

${ }^{1}$ de Moura, K.C.G., Emery, F.S., Pinto, C.N., Pinto, M.C.F.R., Dantas, A.P., Salomão, K., de Castro, S.L., Pinto, A.V. J. Braz. Chem. Soc. 2001, 12, 325-338.

${ }^{2}$ de Moura, K.C.G., Salomão, K., Menna-Barreto, R.F.S., Emery, F.S., Pinto, M.C.F.R., Pinto, A.V., Castro, S.L, Eur. J. Med. Chem. 2004, 39, 639-645.

${ }^{3}$ Silva, R. S. F.; Leitão, G. G.; Brum, T. B.; Lobato, A. P. G.; Pinto, M. C. F. R.; Pinto, A. V. J. Chromatogr. A. 2007, 1151, 197. 